\title{
Applications of Emerging Nanomaterials for Oily Wastewater Treatment
}

\author{
P.S. Goh ${ }^{1}$, C.S. Ong ${ }^{2}$, B.C. $\mathrm{Ng}^{1}$ and A.F. Ismail ${ }^{1}$ \\ ${ }^{1}$ Advanced Membrane Technology Research Centre (AMTEC), Faculty of Chemical and \\ Energy Engineering, Universiti Teknologi Malaysia (UTM), Johor, Malaysia. \\ ${ }^{2}$ Water Desalination and Reuse Center, Division of Biological and \\ Environmental Science and Engineering, King Abdullah University of Science and \\ Technology, Thuwal 23955-6900, Saudi Arabia. \\ Corresponding Author: peisean@petrolem.utm.my
}

\begin{abstract}
The huge volume of oily wastewater produced from the industries has resulted in alarming environmental pollution and resource usage problems. A wide range of conventional and emerging approaches have been established to effectively reduce all these pollutants to an acceptable level to discharge the treated wastewater for reuse. Lately, nanomaterials have shown great potential to address this issue based on their unique and exceptionally properties such as high surface area, superhydrophilicity/hydrophobicity and surface functionalities. This chapter discusses the application of emerging nanomaterials for oily wastewater treatment. The characteristics of these nanomaterials and their hybrid or nanocomposites for oily water treatment are discussed. Finally, a brief future outlook and concluding remarks are presented.
\end{abstract}

Keywords: nanomaterials; oily wastewater; membrane, adsorption 


\section{Introduction}

Water is a critical resource used for economic, social and cultural development. However, with the increase of population and the developments brought by the refineries, petrochemical and transportation industrial revolution, water pollution is an inevitable issue faced by the mankind in this century (Jamaly, Giwa \& Hasan 2015). Currently, one of the most significant waste found in the water sources is oil discharge from oil and gas industries. In general, oily wastewater refers to the wastewater that has mixed with oil with a broad range of concentrations. The oil compounds found in oily wastewater may consist of fats and hydrocarbons as well as petroleum fractions. Many industries produce a huge amount of oily wastewater which have significant adverse impacts and threats to the surrounding environment and human beings due to the presence of hazardous contents in the wastewater. For instance, high consumption of oil and gas in conventional petrochemical refinery industries poses critical environmental issues in the waste disposal management. Oily wastewater which collected from different industrial source may vary in term of its chemical composition, physical characteristics. Hence different treatment designs have been reported based on the required specifications, wastewater's characteristics and pollution parameters. Produced water generated from oil well is known to be one of the most hazardous waste stream if the wastewater is disposed directly without treatment as it contains large quantity of oily pollutants such as dissolved oil, grease, suspended particles, gases and minerals, as well as some insoluble organic substances. The evaporation of these contents may cause air pollution and the penetration of produced water into underground water resources. These pollutants also resulted in several problems in process equipment such as tubular corrosion and scaling formation in heat exchanger. Besides that, the discharge of these oily components also known to affect crop production and destructing the natural landscape.

An appropriate wastewater strategy is required to treat the produced water prior to their disposal or reuse for other purposes. The appropriateness of an identified technology is normally dictated by the characteristics of the oil components in water. Particularly, it is known that the degree of dispersion and the oil droplet stability in water has strong influence in the readiness of separation. The physical characteristics of oil components and their diameter range are tabulated in Table 1. Different treatment methods have been utilized to remove the oil impurities. The treatment processes normally involved the removal of dispersal oil and grease; soluble organic; suspended solid removal, dissolved 
gas such as carbon dioxide; desalination for salt removal and disinfection of microorganisms. Currently, a wide selection of treatment methods has been established for the removal of the oil impurities in order to minimize or prevent the negative impacts of oily wastewater on our environments. Some of the prevailing technologies include membrane filtration, electrochemical treatment, adsorption, floatation and chemical coagulations as well as the hybrid technologies that integrate two or more approaches mentioned above. These technologies are known to serve different purposes in oily wastewater treatment. The primary treatment that consist of gravitational separation and sedimentation normally aims to remove oil and grease components in free oil form and the settlement of some unstable colloidal particles.

Table 1: Physical classification of oil and grease compounds in aqueous medium (Pintor et al. 2016)

\begin{tabular}{|l|l|l|}
\hline Classification & Diameter range & Characteristics \\
\hline Free oil & $>150 \mu \mathrm{m}$ & $\begin{array}{l}\text { Droplets that float on the surface of aqueous } \\
\text { medium due to the density difference of oil and } \\
\text { water }\end{array}$ \\
\hline Dispersed oil & $20-150 \mu \mathrm{m}$ & Droplets stabilized by electric charges \\
\hline Emulsified oil & $<20 \mu \mathrm{m}$ & $\begin{array}{l}\text { Droplets stabilized by the chemical action of } \\
\text { surface active agent }\end{array}$ \\
\hline Soluble oil & $<5 \mu \mathrm{m}$ & $\begin{array}{l}\text { Very fine droplets that dissolve in aqueous } \\
\text { medium }\end{array}$ \\
\hline
\end{tabular}

The emergence of a wide range of nanomaterials rendered with astonishing properties has promised innovative and novel way to treat wastewater hence minimizing the negative impact of the oily wastewater discharge into the water sources (Yu et al. 2017). In term of nanomaterial selection, several kinds of surface wetting properties have been considered to deliver efficient oily wastewater treatment. These surface properties include hydrophobic and oleophilic, hydrophilic and oleophobic, superhydrophilic and superoleophobic, and 
responsive wetting properties. For example, the superoleophobic nanomaterials normally exhibit very high water affinity but extremely low underwater oil adhesion force. As a result, higher water permeation can be obtained and excellent oil repellent can be achieved under water. The surface properties of nanomaterials are known to be affected by their chemical composition and surface roughness. It is also known that superwetting surface can be created by choosing the suitable materials and through various physical or/and chemical treatment (Hou et al. 2017). Hence, various strategies have been developed to synthesize or modify the nanomaterial surfaces in order to obtain desired features like high permeability, self-cleaning ability, catalytic reactivity and ability to mitigate fouling. To date, many efforts have been paid to design and fabricate superwetting nanomaterials for adsorption and membrane-based oil/water separation.

In this chapter, the applications of innovative and novel nanomaterials for oily wastewater treatment are presented. The oily wastewater treatment technologies are first reviewed to provide a general overview on the three major approaches i.e. adsorption, electrochemical and membrane separation. Next, the roles of emerging nanomaterials in reducing the adverse effect of oily wastewater through different approaches are highlighted. Finally, the challenges and opportunities of this particular field for future development are also briefly discussed.

\section{Contemporary Technologies for Oily Wastewater Treatment}

\subsection{Adsorption}

One widely applied technique to remove dissolved organic substances in water is adsorption where the substances are separated from one phase to the surface of another. Adsorption is one of the most frequently used separation processes for the treatment of waste in industry scale. It is a process that involves the adhesion of pollutants onto the surface of a substance without creating a huge amount of hazardous sludge. The adsorbing medium is generally known as the adsorbent whereas the material adsorbed on the surface is known as the adsorbate. Some of the important criteria for the selection of nanomaterials are their accessibility, cost-effectiveness and ability to reduce environmental impact. In term of the mechanisms involved, adsorption can be divided into two, i.e. chemical and physical 
adsorption. The former mechanism involves the chemical reaction where adsorbate is chemically reacted with the surface. On the other hand, the latter involves the attachment of waste substances through physical forces such as hydrogen ponding and dipole-dipole interaction (Kausar et al. 2018). A list of materials has been applied for water remediation. Some naturally found mineral or organic adsorbent shown benefits such as low cost, environmentally friendliness and high oil removal efficiency. The typically required characteristics of adsorbent are high surface area, charge density and hydrophobicity. In term of surface feature, corrugations and hair-like features are also desired to promote oil uptake through capillary forces (Pintor et al. 2016).

Among these materials, activated carbon has been widely used owing to its unique adsorbing properties such as highly porous and large surface area. It has been used for the treatment of petroleum contaminated ground-water and it is known that powdered activated carbon (PAC) is generally more effective than GAC (granular activated carbon). However, despite the efficiency in biochemical oxygen demand (BOD) and COD removal, adsorption based on activated carbon is largely limited by its expensive cost. Furthermore, the high oil concentrations may result in pore clogging and deteriorate the adsorption performance. Clay is another class of material that exhibits promising adsorptive behaviour to remove organic pollutants. The utilization of different clays as adsorbents for the removal of oily components from wastewater has received attentions mainly due to the eco-friendly nature of clay materials. It has been reported that bentonite organo-clay can serve as an effective adsorbent to remove oil from oily waters in a column system in which the capability is $5-7$ times better than that of activated carbon. Over the last few decades, the application of natural fibers for oily wastewater treatment has also been reported (Wahi et al. 2013). Some commonly used natural oil adsorbents are rice husk, sugarcane bagasse and wood residues which are normally formed into sheets, filers or fiber assemblies. These natural adsorbents are derived from the waste hence chemical-free and highlight biodegradable.

Recently, many nanoparticles have been studied for their potential as adsorbents. The nanosized adsorbents are featured by two main properties, i.e. innate surface and external functionalization (Anjum et al. 2016). It is well agreed that the physical and chemical properties of these nanomaterials are also strongly associated to their extrinsic properties such as surface structure and apparent size. The effectiveness of nanoparticles that used for adsorption are influenced by their size and shape, surface chemistry, solubility and 
dispersion state, chemical composition as well as crystallinity. However, for oily wastewater treatment, it is generally required that these nanoparticles should be nontoxic, high adsorption capacity, able to adsorb pollutants in concentration as low as ppb level, easy desorption process and can be recycled for several times without much affecting the overall effectiveness.

\subsection{Electrochemical Separation}

Electrochemical separation of oily waste involves the destabilization the oil emulsion in wastewater through the application of electrical current (Jamaly, Giwa \& Hasan 2015). In fact, electrochemical method has been favourably used in many types of wastewater treatments due to its environmentally friendliness that produces zero or very less secondary pollution. This technique features many interesting properties for oily wastewater treatment such as highly efficient, simple post processing, stable and small footprint (Yang et al. 2015). Electrochemical methods are based on electrochemical oxidation processes using several electrodes. As such, the properties of electrode materials are of crucial importance to determine the efficiency of electrochemical separation. Different types of conductive materials such as iron, aluminium and boron doped diamond have been widely used as the electrode materials. Electroflotation and electrocoagulation are the two common electrochemical methods used for oily wastewater. The principle of electrofloatation lies on the dissociation of water to produce $\mathrm{H}_{2}$ and $\mathrm{O}_{2}$ with low density small bubble particle size that ranges from 20 to $50 \mu \mathrm{m}$. These micro-sized bubbldes can quickly absorb emulsified oil droplet and other suspended waste to form bigger lumps that float to the water surface. Their strong adsorption capacity and floating load capacity of these bubbles can rapidly result in fast flotation separation and effective oil removal (Yang et al. 2015). On the other hand, electrocogulation relies on the production of in situ coagulants from an electrode through the electric current applied to the electrodes. The production of ions is subsequently followed by the electrophoretic concentration that takes place at the anode. These ions are attracted by the colloidal particles and their charges are neutralized to allow coagulation to take place. The hydrogen gas formed at the cathode is then interact with the particles to facilitate the removal of the unwanted materials (Andrade \& Costa Marques 2012). 


\subsection{Membrane Technology}

Membrane technology is a promising solution for oily wastewater treatment due to its advantages such as cost effectiveness, free from chemical additives, modular installation and operation at ambient temperature compared to those conventional methods (Padaki et al. 2015). In a broad term, membrane filtration involves the physical separation of the unwanted impurities from the bulk solutions through a semi-permeable membrane. Depending on the principles of the membrane technology, hydraulic pressure or osmotic pressure is required for the operation of this technology. Different types of pressure driven membrane-based processes such as microfiltration (MF), ultrafiltration (UF), nanofiltration (NF), reverse osmosis (RO) have been widely used to treat oily waste water. Recently, forward osmosis (FO) has also been explored for this purpose. Owing to the modular operations of membrane separation, some of these processes have been integrated to attain higher removal efficiencies. Extensive research in membrane filtration technologies for oily wastewater treatment has been performed over the past 20 years. In general, two broad categories of commercial and lab scaled membranes have been applied for oil removal, i.e. ceramic membranes and polymeric membranes. Polymeric membranes have been substantially used for oily wastewater treatment due to its low cost and easy handling. However, compared to the polymeric membranes, ceramic membranes are known to have greater mechanical and chemical stability as well as better resistance towards membrane fouling. Regardless of the types of membranes used, constant efforts have been made to improve the membrane and membrane system in terms of cost and affordability, energy consumption and sustainability. One promising strategy to achieve these improvement is through the development of advanced membrane materials. The application of nanomaterials in membrane technologies to revolutionize the membrane materials in order to tackle the problems related to oily wastewater has become the major focus of the research community in this field (Ahmadi et al. 2017). One of the most significant breakthrough in polymeric membrane development is the fabrication of mixed matrix membrane (MMM) or thin film nanocomposite (TFN). These nano-enabled membranes are formed by incorporating nanomaterials which act as nanofiller into the polymeric substrate (Lee, Arnot \& Mattia 2011). In MMM, the nanomaterials are commonly embedded and randomly distributed within the polymer matrix through physical mixing prior to the formation of MMM through phase inversion technique. On 
the other hand, in the formation of TFN, the nanomaterials can be selectively introduced into the polymeric supportive substrate or /and within the polyamide selective layer. As such, TFN usually allows higher degree of freedom in term of the nanocomposite membrane design. Figure 1 illustrates the typical procedure used to prepare TFN that is incorporated with silver nanoparticles at the polyamide layer through interfacial polymerization (Yin et al. 2013).

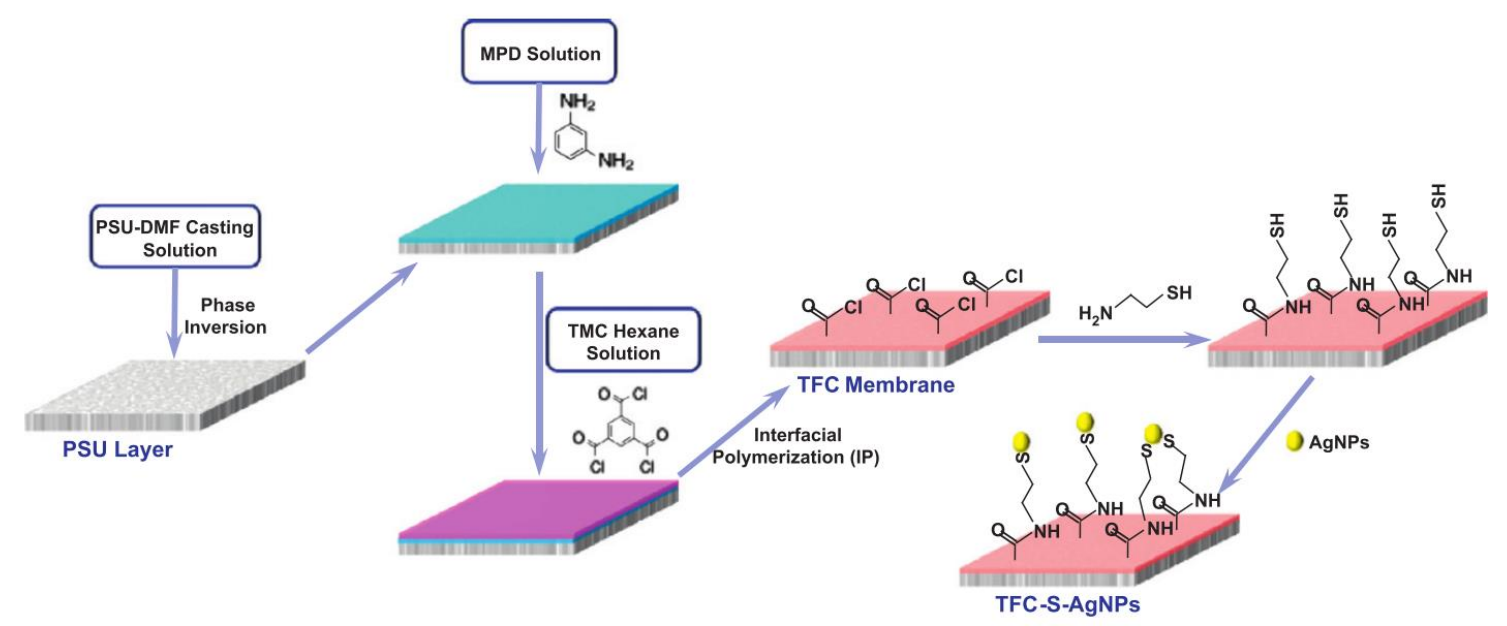

Figure 1: Schematic diagram of TFN that is incorporated with silver nanoparticles at the polyamide layer (Yin et al. 2013)

To date, different types of nanomaterials have been attempted for this purpose. To name a few, carbon-based nanomaterials such as carbon nanotubes (CNTs) and graphene oxide (GO), nanosized metal oxides such as titanium dioxide and silica, silicate-based nanomaterials such as zeolites have been widely applied for the formation of MMM and TFN. The addition of nano-sized materials into the membrane matrix has shown to improve the flux, rejection and also adsorption capacity of these membranes and the usage of these membranes are scattered around the field of water treatment and also gas separation and gas adsorption. It also changes the morphology of the membrane surface and cross section. In general, these nanomaterials possess desired properties such as hydrophilicity, surface charge and mechanical strength to heighten the performance of the resultant nanocomposite membranes. These properties are crucial to resolve some intrinsic membrane problems, i.e. concentration polarisation and fouling. A relatively new class of membrane known as photocatalytic membranes have been recently developed to address the issues related to oily wastewater treatment. Photocatalytic membrane is the integration 
of photocatalysis and membrane technology which can degrade organic pollutants into harmless by-products. This versatile technique has combine the advantages of both photocatalysis and filtration to achieve synergetic effects for oily wastewater treatment. Polymeric membranes such as polysulfone (PSf) and polyvinylidene difluoride (PVDF, ceramic membranes such as alumina and zeolite membranes are commonly used as the host for photocatalyst. Conventional photocatalyst such as Degussa P25 titanium dioxide has been commonly incorporated into the MMM to form photocatalytic membranes with ability to degrade the oil molecules hence reduce the fouling tendency. Moreover, when the photocatalyst is introduced onto the ceramic support or dispersed into the membrane matrix, they can be physically retained and eliminate the step of photocatalyst recovery from the aqueous suspension (Subramaniam et al. 2016).

\section{Roles of Nanomaterials in Oily Wastewater Treatment}

\subsection{Adsorption}

Zhang et al. prepared $\mathrm{Fe}_{3} \mathrm{O}_{4}$ magnetic nanoparticles by a coprecipitation method and followed by surface coating with silica and 3-aminopropyltriethoxysilane (APTES). The resultant nanocomposite adsorbent was then supported onto quaternized chitosan (QC) for adsorption of oil. QC is known to demonstrate permanent cationic charges hence could establish electrostatic interaction with negatively charged oil droplets (Zhang et al. 2017). Owing to the excellent adsorptive properties, the adsorbent exhibited promising oil removal capability at various $\mathrm{pH}$ conditions where under both neutral and acidic conditions, the water transmittance of $98 \%$ was achieved at $34 \mathrm{mg} / \mathrm{L}$ and under alkaline conditions, water transmittance of $98 \%$ was achieved with dosage of $38 \mathrm{mg} / \mathrm{L}$. Importantly, the magnetic adsorbent shown good reusability for practical application where it still exhibited god performance of more than $90 \%$ water transmittance after 8 cycles of operations. Lu et al. also synthesized $\mathrm{pH}$-sensitive and recyclable silica-coated $\mathrm{Fe}_{3} \mathrm{O}_{4}$ magnetic nanoparticles using dense liquid silica coating method (Lü et al. 2017). The oil-water separation mechanism proposed by the authors pointed out that both electrostatic interaction and interfacial activity has significant roles in the oil-water separation. As shown in Figure 2, under acidic condition, the magnetic nanoparticles adsorbed to the negatively charged oil droplets hence facilitate the flocculation of the oil droplets via electrostatic interaction thus can be easily separated through physical magnetic separation. On the other hand, under the 
neutral condition, the magnetic nanoparticles served as non-ionic surfactant and accumulated at the interface of oil-water whereas under alkaline condition, the silica coated nanoparticles were detached from each other due to the electrostatic repulsion. These interesting characteristics of the magnetic nanopartciles have allowed the recycling of this material based on its $\mathrm{pH}$ sensitivity where it can be used as an effective adsorbent to remove emusified oil droplets under acidic and neutral conditions, and can be recycled in alkaline solution through simple rinsing.

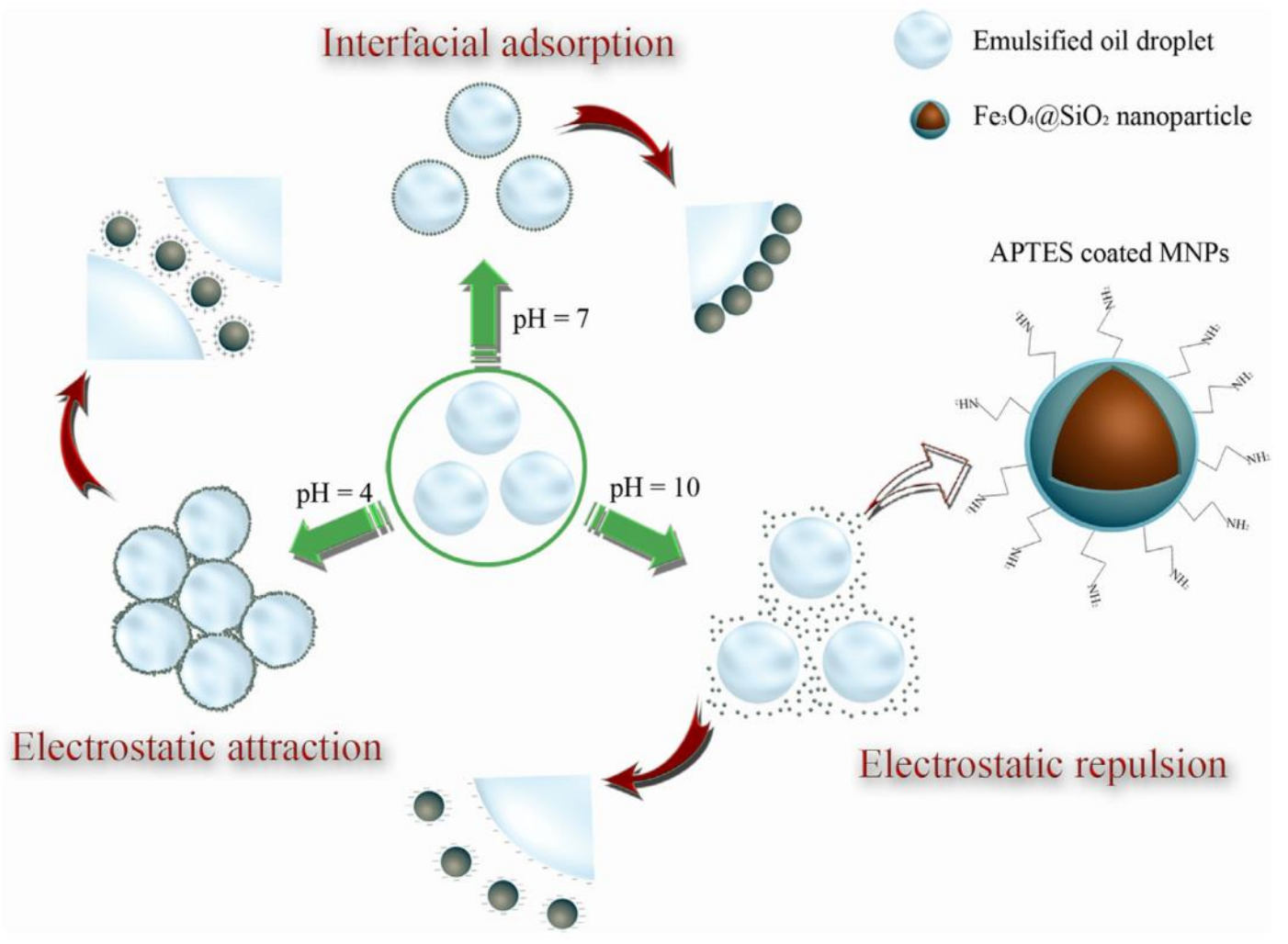

Figure 2: Schematic illustration of the interaction mechanism between the silica coated magnetic nanoparticles and emulsified oil droplets (Lü et al. 2017).

In a recent study conducted by Song et al., bismuth vanadate $\left(\mathrm{BiVO}_{4}\right)$ nanoparticle with sunlight responsive self-cleaning properties has been applied for the synthesis of underwater superoleophobic mesh (Song et al. 2017). Compared to conventionally used $\mathrm{TiO}_{2}$, the unique optical and electrical properties of $\mathrm{BiVO}_{4}$ allow the photocatalytic activity to be activated under visible light. Owing to the excellent underwater superoleophobicity, 
the water in the oil/water mixture can penetrate the mesh easily hence oil droplets can be efficiently removed. A variety of oil/water mixtures such as hexane, diesel, dichloroethane and chloroform have been successfully separated. Furthermore, the adsorption capability can be easily recovered under the irradiation of visible light due to the photocatalytic activity demonstrated by $\mathrm{BiVO}_{4}$ to degrade the organic compounds.

\subsection{Membrane technology}

One of the most critical challenges in membrane processes, particularly the pressure driven processes, is the membrane fouling issue caused by the deposition of oil droplets and soluble organics onto the membrane surfaces. The plugging of the membrane pores is directly associated to the deterioration of membrane performance in in term of the water productivity. Commonly, membrane cleaning is necessary to recover the membrane flux hence impose additional chemical cost to the overall process. Membrane fouling also unfavourably results in the shortening of membranes' life span. In order to combat this primary challenge, many efforts have been devoted to modify and improve the properties of the membrane materials. Different approaches and strategies and have developed to mitigate fouling during operation. These include cross-linking, polymer blending and incorporation of inorganic nanomaterial fillers. Among these approaches, incorporation of hydrophilic nanomaterials has attracted tremendous attentions of the research community due to its effectiveness and feasibility to enhance the performance. In general, upon the introduction of hydrophilic nanomaterials, the resultant nanocomposite membranes demonstrate much improved hydrophilicity hence can easily reject the hydrophobic oil droplet found in the oily wastewater based on the repulsive force formed between the oil molecules and membrane surfaces.

Kusworo et al. compared the performance of PES membrane incorporated with $\mathrm{ZnO}$ and $\mathrm{SiO}_{2}$ for produced water treatment and it was found that the former was better in terms of the permeate flux (Kusworo, Qudratun \& Utomo 2017). Permeate flux improvement up to $200 \%$ and pollutant rejection efficiency of $16-18 \%$ were observed in the nanocomposite membrane. The oil droplet removal efficiency was much better compared to that of neat PES due to the improved hydrophilicity of the membrane as evidenced from the decrease in surface contact angle. Additionally, lesser foulant deposition was observed on the 
membrane surface compared to that of neat PES membrane. Saadati et al. prepared PSf/pebax MMMs with different loadings of functionalized multi-walled CNTs (MWCNT) for the separation of oil/water emulsion (Saadati \& Pakizeh 2017). By incorporating 2 wt\% of MWCNT into the polymeric matrix, oil rejection has been improved by $8 \%$ at transmembrane pressure of 10 bar compared to that of neat PSf/pebax membranes. The increment was mainly ascribed to the increase in hydrophilicity upon the addition of functionalized MWCNT to increase the water permeation through the membrane. Additionally, the reduction in membrane pore size and porosity upon the addition of MWCNT with loading of more than $0.5 \mathrm{wt} \%$ also led to favourably increased oil rejection. MMMs consisted of dual-nanofillers of hydrous manganese oxide (HMO) and titanium dioxide $\left(\mathrm{TiO}_{2}\right)$ at different have also been reported to improve the properties of polyethersulfone (PES) membrane for oil-water separation (Lai et al. 2017). It was observed that the incorporation of the dual-nanofiller, particularly with higher amount of HMO used, has enhanced the surface hydrophilicity of the membranes. This improvement can be attributed to the presence of huge amount -OH functional groups found in HMO compared to $\mathrm{TiO}_{2}$. The incorporation of hydrophilic nanomaterials has affected the solvent-non solvent exchange rate during the formation of membrane through phase inversion technique, hence prompted the formation of more long finger-like structure. The favourable changes in the cross sectional morphology and surface hydrophilicity have shown positive effect on the water flux enhancement as lower transport resistance for water molecules was anticipated. In this study, the authors reported two best MMMs that consisted of $\mathrm{HMO}$ and $\mathrm{TiO}_{2}$ in the ratio of $0.75: 0.25$ and $0.25: 0.75$. These membranes have achieved $31.73 \%$ and $26.41 \%$ higher water flux, respectively as compared to the neat PES membrane without compromising the oil removal performance. Additionally, lower flux decline was observed due to the improved surface resistance against fouling. Consequently, these samples can be used for longer life span and hold good potential for long-term operation. Figure 3 displays the antifouling properties of membrane by subjecting the membrane to oil contamination and followed by simple water cleaning for two cycles. It is found that the oil stain was easily removed from the membrane surface in comparison to the control membrane due to the presence of hydrophilic nanofillers. 


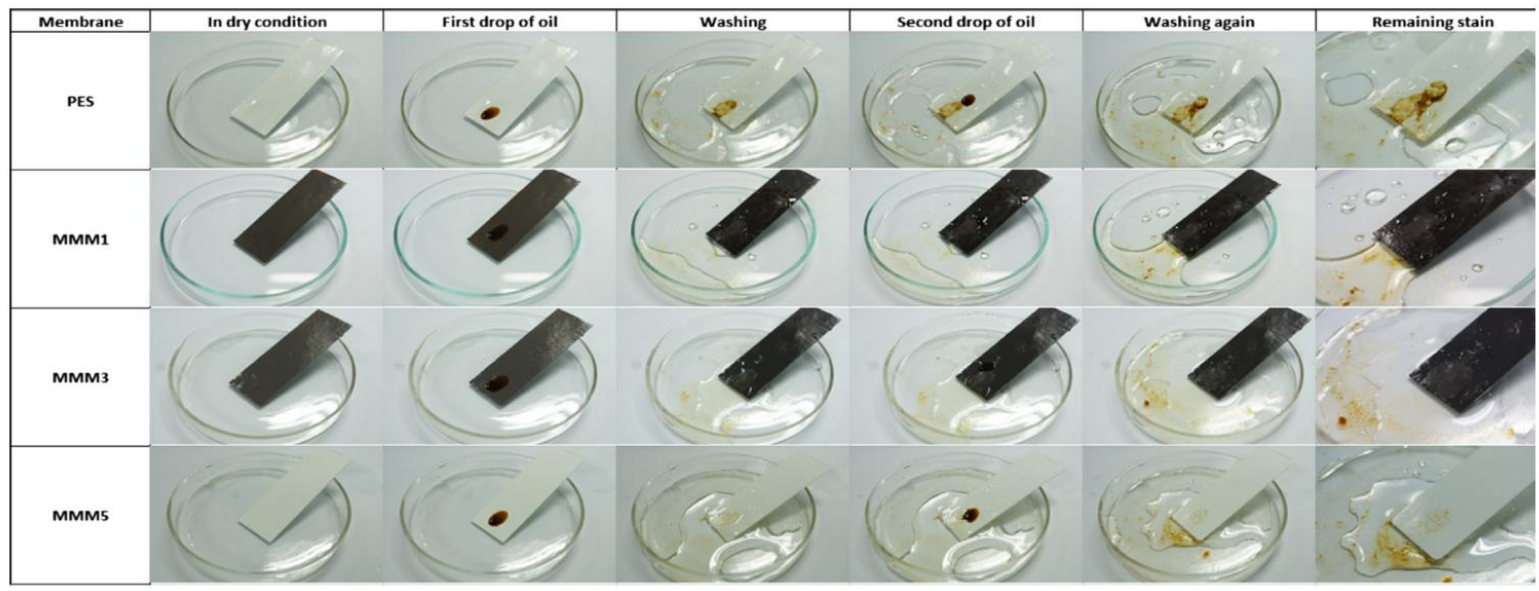

Figure 3: Antifouling studies of the MMMs that involved membrane cleaning with simple water rinsing (Lai et al. 2017).

In term of photocatalytic membranes, study done by Ong et al. showed that $\mathrm{TiO}_{2}$ incorporated PVDF membranes were able to treat cutting oil effluent effectively, where a treatment efficiency of more than $90 \%$ was achieved (Ong et al. 2015). They found that the addition of $\mathrm{TiO}_{2}$ with loading of $2 \mathrm{wt} \%$ has contributed to the enhanced membrane properties by increasing the surface hydrophilicity, pore size and surface roughness of the resultant nanocomposite membranes. However, when the loading of $\mathrm{TiO}_{2}$ was further increased above $2 \mathrm{wt} \%$, agglomeration of nanoparticles took place and deteriorated the performance of the membranes. As shown in the scanning electron microscope (SEM) images in Figure 4, the surface and cross-sectional morphology of the resultant MMM hollow fiber membranes was not significantly influenced by the addition of the photocatalyst. However, at higher concentration, agglomeration (white circle) was observed and resulted in the formation of voids which tend to compromise the oil rejection properties. Another study on oily wastewater done by Rusli et al. showed an oil removal efficiency of $60 \%$ within 6 hours of treatment using a hybrid $\mathrm{PVDF} / \mathrm{TiO}_{2}$ membrane where the photocatalyst was hot pressed onto a PVDF flat sheet membrane for better adhesion (Rusli et al. 2016). 

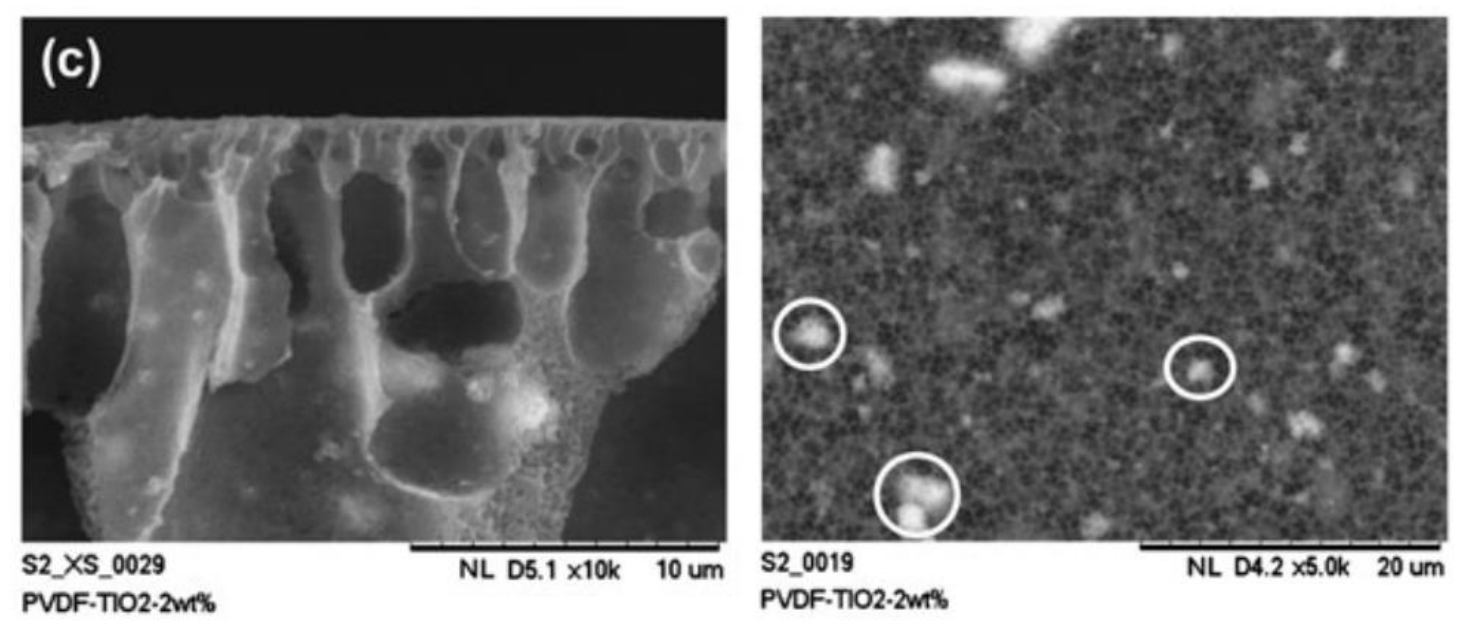

Figure 4: SEM images (cross-section and outer surface) of PVDF membranes with 2 wt\% of $\mathrm{TiO}_{2}$ (Ong et al. 2015)

\subsection{Hybrid Technology}

Despite the maturity of conventionally used technologies for oily wastewater treatment, the feasibility of these technologies for large scale treatment is always restricted by their limitations such as high energy consumption, cost and low efficiency. To counter these shortages, combination of conventional processes such as coagulation, adsorption and ion exchange (hybrid processes) with membrane technology demonstrate somes benefits and improvements such as improving quality of the treated water, minimizing fouling of the membranes, energy savings hence reduce the operating cost of the treatment plants. Rasouli et al. performed oily wastewater treatment by hybrid adsorption-MF process using powdered activated carbon (PAC), natural zeolite powder and ceramic membranes (Rasouli, Abbasi \& Hashemifard 2017). In their study, PAC and natural zeolite powder by concentrations of $100-800 \mathrm{mg} / \mathrm{L}$ have been used as adsorbing agent in in-line adsorptionMF process and they reported the increase in total organic carbon rejection in all concentration of PAC for all membranes when compared to MF process alone.

\section{Conclusion and Future Outlook}

Oily wastewater is a severe issue before it is disposed to the water sources in a manner that does not harm to the mankind and environments. Due to the challenges encountered in oily 
wastewater treatment, many techniques as well as the combinations of different techniques have been developed to remediate the negative impacts caused by this waste. The development and application of advanced functional nanomaterials for oily wastewater treatment has attracted significant interest. In order to efficiently treat stable oil-water emulsion and separate relatively small oil droplets from their bulk solution, numerous studies have been dedicated to design novel functional nanomaterials for oily wastewater separation. Recent advances in the development of nanomaterials have allowed some critical underlying issues related to the existing technologies to be resolved. For instance, the severe membrane fouling caused by the surfactant adsorption and/or pore plugging by oil droplets can be tackled by incorporating functional nanomaterials to obtained the desired properties for oily wastewater treatment. Nevertheless, despite the promising results showed by these nanomaterials in heightening the performances of various commonly used treatment technologies, the complicated issues regarding the oil recovery and the recycling of nanomaterials are a subject to be studied in depth in the economic point of view. In term of adsorption process, it is expected that the development of magnetic nanomaterials can serve as a good option due to its recyclability. Besides that, the embedment of nanomaterials within polymeric matrix also promises the sustainability of this material as the leaching of nanomaterials can be prevented. In term of adsorption, it is anticipated that nanomaterials with switchable wettability will be the future trend of the research. Nanomaterials with responsive wettability that can be altered from wetting to antiwetting or vice versa can offer a significant improvement for controllable oily wastewater separation, particularly in the harsh and complex environmental condition. Although current some efforts have been made to alter the superwettability of the nanomaterial such as CNT film using UV irradiation and dark storage, the recovery time of the switching requires one day. Hence more smart nanomaterials need to be explored to accelerate the switching period for practical usage (Yang et al. 2017). In view of the advantages and limitations of the standalone technologies mentioned above, the hybrid technology that consists of pretreatment techniques such as photocatalytic reactor, gravitational separation, flocculation and adsorption prior to membrane technology will be advantageous to resolve the challenging treatment process. Undeniably, owing to their unique and unprecedented properties, various nanomaterials have found their important roles in different oily wastewater treatment technologies. With more advances and innovations made in this field, it is expected that more emerging and potential nanomaterials will be developed to deal with a large variety of industrial oily wastewater. 


\section{Acknowledgement}

The authors would like to acknowledge the financial support provided by Ministry of Higher Education under HiCOE Grant (4J182) and Universiti Teknologi Malaysia under Research University Grant (18H35).

\section{References}

Ahmadi, A., Qanati, O., Seyed Dorraji, M.S., Rasoulifard, M.H. \& Vatanpour, V. 2017, 'Investigation of antifouling performance a novel nanofibrous S-PVDF/PVDF and SPVDF/PVDF/GO membranes against negatively charged oily foulants', Journal of Membrane Science, vol. 536, no. 1, pp. 86-97.

Andrade, A. \& Costa Marques, M.R. da 2012, 'Electrolytic Treatment of Wastewater in the Oil Industry', New Technologies in the Oil and Gas Industry, pp. 3-28.

Anjum, M., Miandad, R., Waqas, M., Gehany, F. \& Barakat, M.A. 2016, 'Remediation of wastewater using various nano-materials', Arabian Journal of Chemistry.

Hou, K., Zeng, Y., Zhou, C., Chen, J., Wen, X., Xu, S., Cheng, J., Lin, Y. \& Pi, P. 2017, 'Durable underwater superoleophobic PDDA/halloysite nanotubes decorated stainless steel mesh for efficient oil-water separation', Applied Surface Science, vol. 416, pp. 344-52.

Jamaly, S., Giwa, A. \& Hasan, S.W. 2015, 'Recent improvements in oily wastewater treatment : Progress, challenges , and future opportunities', Journal of Environmental Sciences, vol. 37, pp. 15-30.

Kausar, A., Iqbal, M., Javed, A., Aftab, K., Nazli, Z.-H., Bhatti, H.N. \& Nouren, S. 2018, 'Dyes adsorption using clay and modified clay: A review', Journal of Molecular Liquids, vol. 256, pp. 395-407.

Kusworo, T.D., Qudratun \& Utomo, D.P. 2017, 'Performance evaluation of double stage process using nano hybrid PES/SiO2-PES membrane and PES/ZnO-PES membranes for oily waste water treatment to clean water', Journal of Environmental Chemical Engineering, vol. 5, no. 6, pp. 6077-86.

Lai, G.S., Yusob, M.H.M., Lau, W.J., Gohari, R.J., Emadzadeh, D., Ismail, A.F., Goh, P.S., Isloor, A.M. \& Arzhandi, M.R.-D. 2017, 'Novel mixed matrix membranes incorporated with dual-nanofillers for enhanced oil-water separation', Separation and Purification Technology, vol. 178.

Lee, K.P., Arnot, T.C. \& Mattia, D. 2011, 'A review of reverse osmosis membrane materials 
for desalination-Development to date and future potential', Journal of Membrane Science, vol. 370, no. 1-2, pp. 1-22.

Lü, T., Zhang, S., Qi, D., Zhang, D., Vance, G.F. \& Zhao, H. 2017, 'Synthesis of pHsensitive and recyclable magnetic nanoparticles for efficient separation of emulsified oil from aqueous environments', Applied Surface Science, vol. 396, pp. 1604-12.

Ong, C.S., Lau, W.J., Goh, P.S., Ng, B.C. \& Ismail, A.F. 2015, 'Preparation and characterization of PVDF-PVP-TiO<inf $>2</$ inf $>$ composite hollow fiber membranes for oily wastewater treatment using submerged membrane system', Desalination and Water Treatment, vol. 53, no. 5.

Padaki, M., Murali, R.S., Abdullah, M.S., Misdan, N., Moslehyani, A., Kassim, M.A., Hilal, N. \& Ismail, A.F. 2015, 'Membrane technology enhancement in oil - water separation . A review', Des, vol. 357, pp. 197-207.

Pintor, A.M.A., Vilar, V.J.P., Botelho, C.M.S. \& Boaventura, R.A.R. 2016, 'Oil and grease removal from wastewaters: Sorption treatment as an alternative to state-of-the-art technologies. A critical review', Chemical Engineering Journal, vol. 297, pp. 229-55.

Rasouli, Y., Abbasi, M. \& Hashemifard, S.A. 2017, 'Oily wastewater treatment by adsorption-membrane filtration hybrid process using powdered activated carbon, natural zeolite powder and low cost ceramic membranes', Water Science and Technology, vol. 76, no. 4, pp. 895-908.

Rusli, U.N., Alias, N.H., Shahruddin, M.Z. \& Othman, N.H. 2016, 'Photocatalytic degradation of oil using polyvinylidene fluoride/titanium dioxide composite membrane for oily wastewater treatment', MATEC Web of Conferences, vol. 69.

Saadati, J. \& Pakizeh, M. 2017, 'Separation of oil/water emulsion using a new PSf/pebax/FMWCNT nanocomposite membrane', Journal of the Taiwan Institute of Chemical Engineers, vol. 71, pp. 265-76.

Song, S., Yang, H., Zhou, C., Cheng, J., Jiang, Z., Lu, Z. \& Miao, J. 2017, 'Underwater superoleophobic mesh based on BiVO4nanoparticles with sunlight-driven self-cleaning property for oil/water separation', Chemical Engineering Journal, vol. 320, pp. 342-51.

Subramaniam, M.N., Goh, P.S., Ismail, A.F. \& Lau, W.J. 2016, 'Effect of titania nanotubes on the flux and separation performance of polyethersulfone membranes', IOP Conference Series: Earth and Environmental Science.

Wahi, R., Abdullah, L., Shean, T., Choong, Y. \& Ngaini, Z. 2013, 'Oil removal from aqueous state by natural fibrous sorbent An overview.pdf', Separation ans Purification Technology, vol. 113, pp. 51-63. 
Yang, M., Li, Q., Yin, X., Zhai, L. \& Jing, B. 2015, Field Test of Electrochemical Degradation and Oil-Removal Technology Used the Treatment of Wastewater Containing Polymer in Offshore Oilfield, no. December, pp. 1409-15.

Yang, W., Li, J., Zhou, P., Zhu, L. \& Tang, H. 2017, 'Superhydrophobic copper coating: Switchable wettability, on-demand oil-water separation, and antifouling', Chemical Engineering Journal, vol. 327, pp. 849-54.

Yin, J., Yang, Y., Hu, Z. \& Deng, B. 2013, 'Attachment of silver nanoparticles ( AgNPs ) onto thin- fi lm composite ( TFC ) membranes through covalent bonding to reduce membrane biofouling', Journal of Membrane Science, vol. 441, pp. 73-82.

Yu, L., Ruan, S., Xu, X., Zou, R. \& Hu, J. 2017, 'One-dimensional nanomaterial-assembled macroscopic membranes for water treatment', Nano Today, vol. 17, pp. 79-95.

Zhang, S., Lü, T., Qi, D., Cao, Z., Zhang, D. \& Zhao, H. 2017, 'Synthesis of quaternized chitosan-coated magnetic nanoparticles for oil-water separation', Materials Letters, vol. 191, pp. 128-31. 